\title{
THE ULTRAVIOLET SURFACE BRIGHTNESS OF REFLECTION NEBULAE AND THE ALBEDO OF INTERSTELLAR GRAINS
}

\author{
CHARLES F. LILLIE \\ Dept. of Physics and Astrophysics, Laboratory for Atmospheric and Space Physics, The University of \\ Colorado, Boulder, Colo., U.S.A. \\ and \\ ADOLF N. WITT \\ Dept. of Physics and Astronomy, Ritter Astrophysical Research Center, The University of Toledo, \\ Toledo, Ohio, U.S.A.
}

\begin{abstract}
Satellite observations of the Merope and Barnard's Loop nebulae in the 1050 to $4250 \AA$ spectral region show that the increase in the albedo of interstellar grains below $2000 \AA$ occurs in individual reflection nebulae as well as in the general interstellar medium. Evidence for differences in the composition and size distribution of grains due to variations in the local radiation field is presented.
\end{abstract}

\section{Introduction}

The scattering properties of interstellar dust grains in the ultraviolet are important input parameters for models of interstellar and circumstellar clouds. Recently, we have determined the average albedo of particles in the general interstellar medium in the $1500-4250 \AA$ region (Witt and Lillie, 1971). We find the albedo of interstellar grains (see Figure 4) is fairly constant at $a \approx 0.5$ from $3000-4000 \AA$, drops rapidly to a minimum $(a \approx 0.1)$ at $2200 \AA$, then rises to $a \approx 1.0$ at $1500 \AA$. We tentatively attribute this to the presence of three different types of particles in the interstellar medium: a few micron-sized, ice-coated particles which dominate scattering in the visible and near ultraviolet; numerous sub-micron, graphite-like grains which absorb very strongly near $2200 \AA$; and large numbers of submicron, silicate-like grains which dominate scattering in the far ultraviolet (Witt, 1973).

The rapid increase in particle albedo below $2000 \AA$ may be questioned because we did not correct for the possible contribution of faint, blue, horizontal-branch stars at high galactic latitudes (Hills, 1972); also Henry and Carruthers (1970) were unable to detect Barnard's Loop Nebula in the 1230-2100 A spectral region with a rocket-borne electronographic camera. To answer these questions, and to search for variations in the scattering properties of grains in different radiation fields, we have begun the analysis of ultraviolet surface brightness measurements of reflection nebulae obtained with the Orbiting Astronomical Observatory OAO-2. In this paper we report the preliminary results of this investigation for the Merope and Barnard's Loop Nebulae.

\section{The Observational Material}

All of the observations discussed in this paper were obtained with the four stellar 


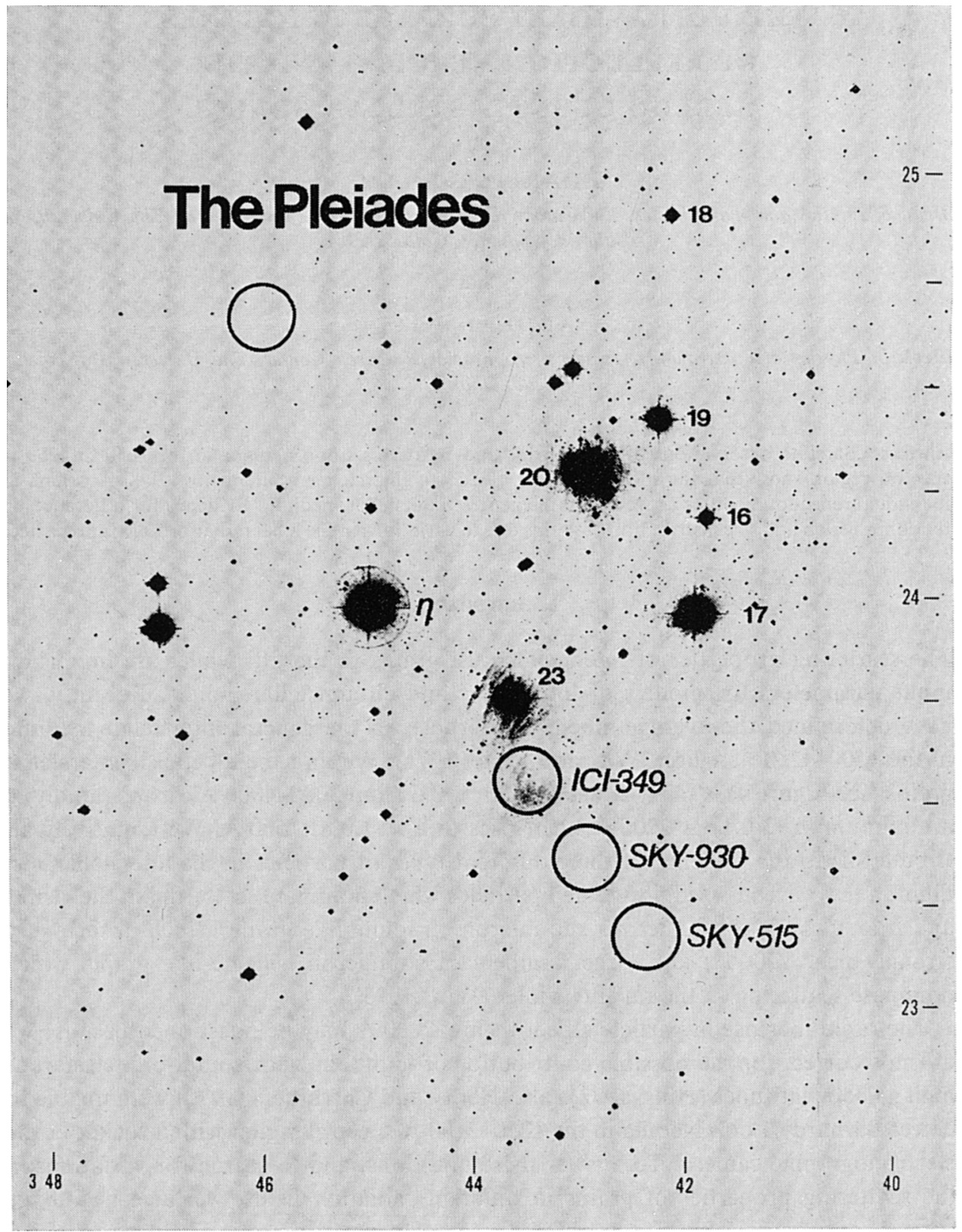

Fig. 1. Location of areas in the Merope Nebula observed with OAO-2 shown on a reproduction from the red plate of the Palomar Sky Atlas. The circles indicate the size of the diaphragm used.

photometers of the University of Wisconsin Experiment Package (WEP) on OAO-2. The instrumentation and operation of this experiment have been described in detail by Code et al. (1970). Each of the stellar photometers is located at the focus of an 


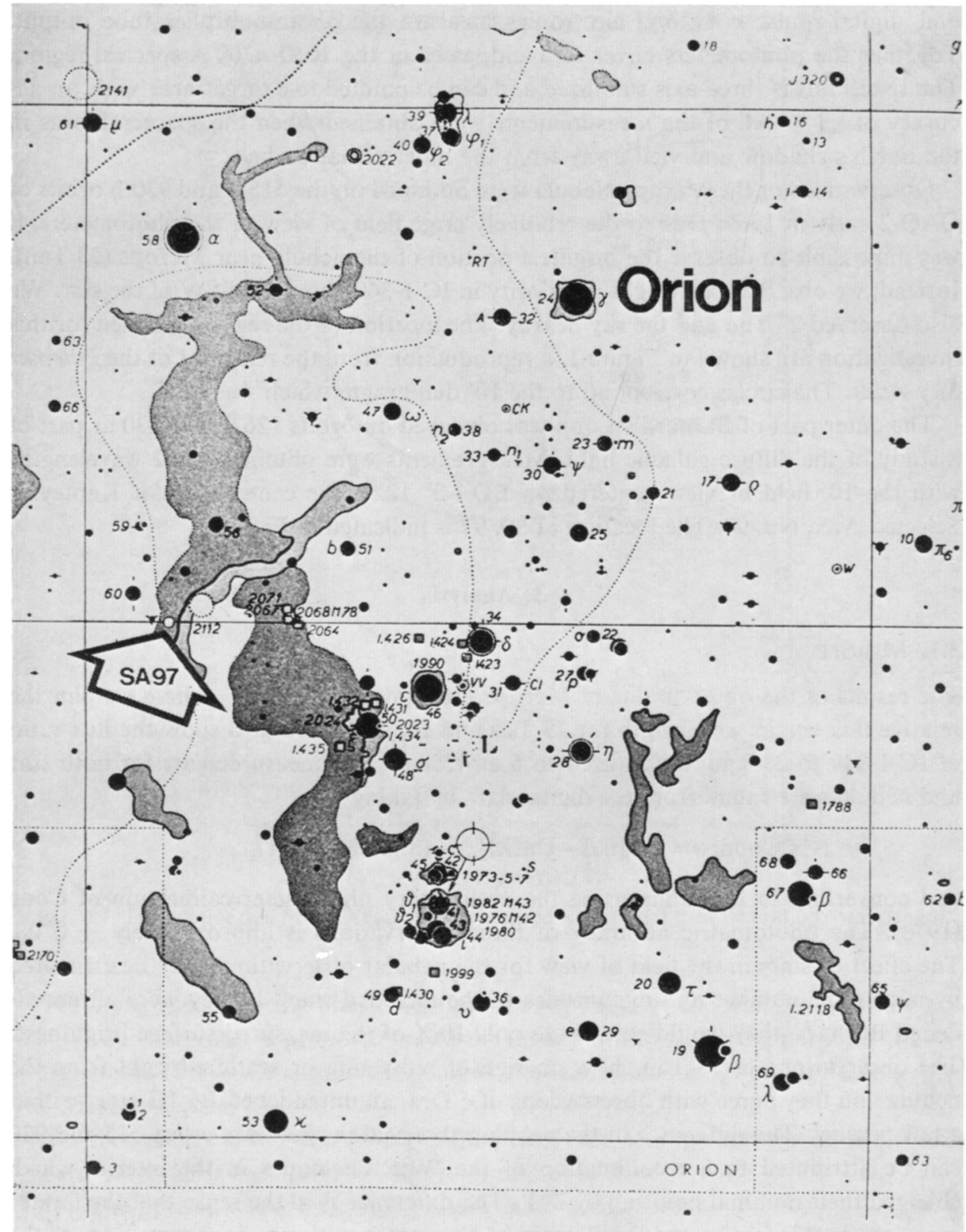

Fig. 2. Location of Kapteyn's Selected Area 97 with respect to Barnard's Loop shown on a reproduction from the Atlas Coeli.

eight-inch, off-axis, parabolic mirror and consists of an aperture mechanism; a fiveposition filter wheel with three medium band interference filters, a dark slide, and a radioactive calibration source; a Fabry lens; and a photomultiplier tube. Both analog 
and digital (pulse counting) electronics measure the photomultiplier tube output. Together the photometers cover 12 bandpasses in the $1050-4300 \AA$ spectral region. The spacecraft is three-axis stabilized and can be pointed to a target area with an accuracy of $\pm 1^{\prime}$. All of the measurements were obtained when the spacecraft was in the Earth's shadow and well away from the inner radiation belt.

Observations of the Merope Nebula were obtained on the 515th and 930th orbits of OAO-2 early in 1969. Due to the relatively large field of view of the photometers, it was impossible to observe the brightest portion of the nebula near Merope (23 Tau). Instead, we observed the bright nebulosity in IC 1-349 about 15' SSW of the star. We also observed 23 Tau and the sky nearby. The location of the regions selected for this investigation are shown in Figure 1, a reproduction from the red print of the Palomar Sky Atlas. The circles correspond to the $10^{\prime}$ diaphragm which was used.

The outer part of Barnard's Loop was observed on orbits 1267 and 4330 as part of a study of the diffuse galactic light. Measurements were obtained at 12 wavelengths with the $10^{\prime}$ field of view centered on BD $+0^{\circ} 1227$, the central star of Kapteyn's Selected Area No. 97. The location of SA 97 is indicated in Figure 2.

\section{Analysis}

\subsection{MEROPE}

The results of the observations of Merope are shown in Figure 3, where we plot the relative flux versus wavelength for 23 Tau and IC 1-349. We also show the flux ratio of IC 1-349 to $23 \mathrm{Tau}$, normalized to 5 at $4250 \AA$. The measurements for both star and nebula were found from the digital data by taking

$$
\text { Net Counts }=(\text { Signal }- \text { Dark })_{\text {Star }}-(\text { Signal }- \text { Dark })_{\text {Sky }}
$$

and converting to flux units using the preliminary photometer calibration of Code (1970). The photometric accuracy of these observations is approximately $\pm 00^{m} 03$. The effect of stars in the field of view for the nebular observations may be estimated by noting the number and magnitudes of the included stars. If they were all unreddened B6 stars, they would contribute only $10 \%$ of the measured surface brightness. The observations of 23 Tau show no sign of reddening or scattered light from the nebula and they agree with observations of $\zeta$ Dra, an unreddened B6 III star, within a few percent. The difference in the nebular observations between orbits 515 and 930 can be attributed to a recollimation of the WEP telescopes in the interim which changed their nominal pointing by $2-3^{\prime}$. The difference is in the sense that the fainter area (orbit 930) is also redder. This is in agreement with previous measures of the Merope Nebula (Collins, 1937; O'Dell, 1965). The $U-B$ colors which we form from our data are in good quantitative agreement with previous measures (O'Dell, 1965).

Because a complete analysis of these data is a complicated problem in radiative transfer involving assumptions about the geometry of the nebula and the reddening curve within it, we confine ourselves at this time to qualitative statements about the nebula. 
THE ULTRAVIOLET REFLECTIVITY OF THE MEROPE NEBULA

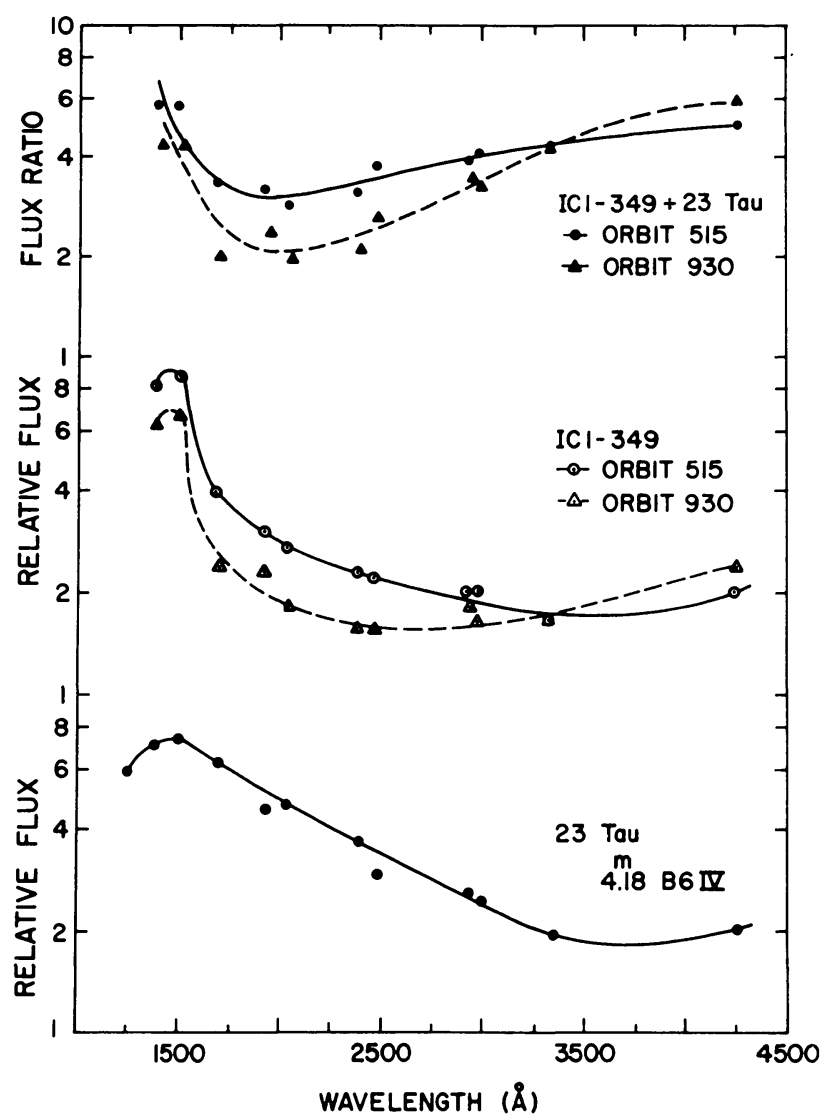

Fig. 3. The relative flux from 23 Tau and the Merope Nebula and their flux ratio vs wavelength.

Providing the scattering phase function does not change too drastically with wavelength, our flux ratio is proportional to the albedo of the scattering particles at every wavelength. This is the case in the general interstellar medium, and we assume it for the Merope nebula.

We then compare our albedo curve (flux ratio) for Merope and the albedo for the general interstellar medium (see Figure 4) and note that:

(1) The upturn below $2000 \AA$ is present in both cases, and

(2) The absorption feature at $2200 \AA$ is less pronounced or missing in the nebula.

This seems clear evidence that the upturn in the albedo of interstellar particles is real and not due to some unexpected source of far ultraviolet radiation (such as horizontal-branch stars or the extragalactic sky background). The lack of a clear absorption feature at $2200 \AA$ seems to indicate a deficiency in the number of absorbing graphite-like grains in the vicinity of 23 Tau due to evaporation, radiation damage, or particle size segregation in the local radiation field. 


\section{THE ALBEDO OF DUST IN BARNARD'S LOOP}

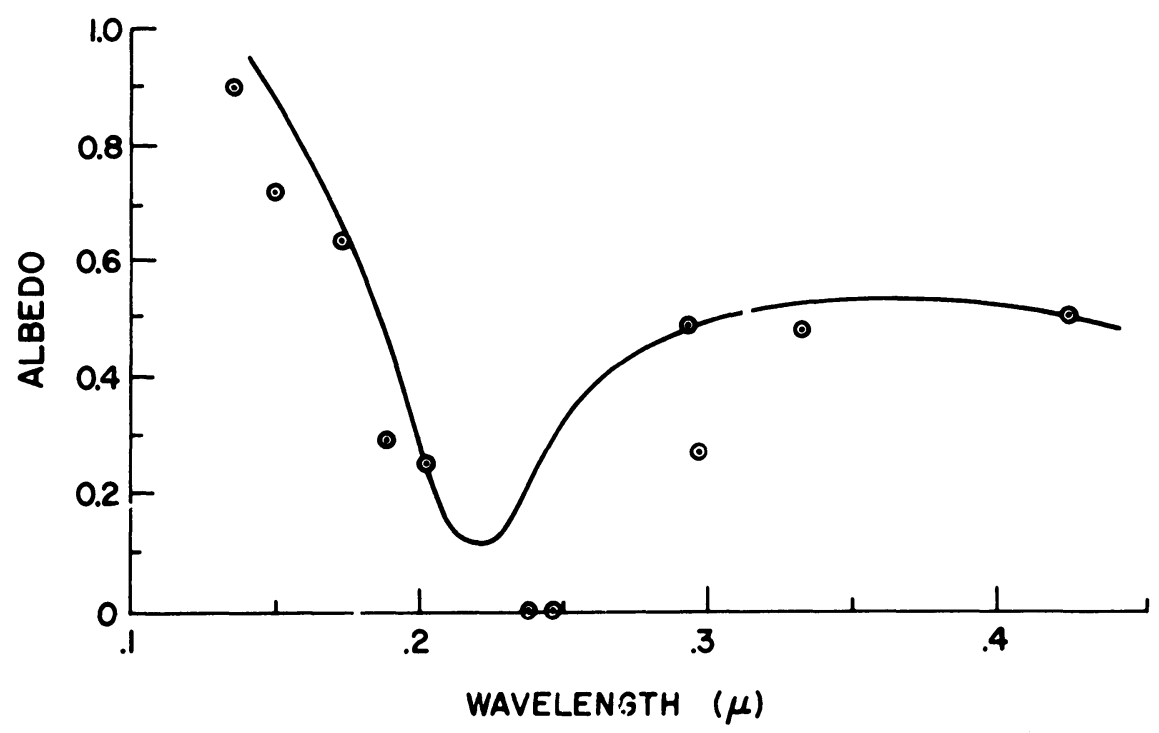

Fig. 4. The albedo of dust in Barnard's Loop (open circles) compared with the albedo of dust particles in the general interstellar medium (solid line).

\subsection{BARNARD'S LOOP}

\subsubsection{OAO Measurements}

Selected Area 97 is located in the outer part of Barnard's Loop. Detailed starcounts exist for it in the Harvard-Groningen Durchmusterung of Selected Areas and the Mount Wilson Catalogue of Photographic Magnitudes in Selected Areas 1-139. These starcounts provide an excellent means of determining the contribution of integrated starlight to the measured surface brightness. In this case, the principal contribution is due to the central star itself, and its ultraviolet brightness can be inferred from its visual magnitude, spectral type, and OAO observations of stars of similar spectral characteristics. The MK spectral type of $\mathrm{BD}+0^{\circ} 1227$ is $\mathrm{B} 9.5 \mathrm{~V}$, as determined from grating spectra obtained with the Cassegrain spectrograph on the 40-in. reflector of the Ritter Astrophysical Research Center. The UBV magnitudes were measured with a photometer on a 16-in. telescope at Kitt Peak National Observatory and found to be: $V=$ 6.91, $B-V=-0.01, U-B=-0.13$.

The other sources of the measured surface brightness are the zodiacal light and light scattered by dust particles in Barnard's Loop and along the line of sight. The zodiacal light contribution can be determined quite easily from the tables of Smith et al. (1965) and an investigation of the ultraviolet spectrum of the zodiacal light by Lillie (1971).

The albedo of the interstellar grains may be found by taking the ratio of the scat- 
tered light intensity to that of the illuminating starlight, assumed to be that of a B6V star. The choice of a spectrum was based on an observational study of the interstellar radiation field by Lillie (1968) who found its spectrum to be that of a B7V + G8V combination in the vicinity of the Sun. In the vicinity of the Orion Association, however, we expect the illuminating starlight to have a somewhat earlier spectral type.

In Figure 4 we have plotted the ratio of scattered light to illuminating starlight for SA 97 normalized to 0.5 at $4250 \AA$ at 11 wavelengths (open circles). For comparison, we show the albedo of dust in the general interstellar medium (solid line) from a previous study (Witt and Lillie, 1971). The agreement over the entire wavelength range is quite satisfactory, although there seems to be somewhat more absorption at $2200 \AA$ than we observe for the average field. We consider these observations of Barnard's Loop an independent confirmation of our previous results. We conclude that the dust particles in the Barnard's Loop nebula have approximately the same scattering properties as particles in the general interstellar medium.

\subsubsection{Other Observations}

The Barnard's Loop region has been observed in the ultraviolet by at least two other space experiments: from the Gemini 11 spacecraft with a hand-held camera with an objective prism which viewed the $2200-4900 \AA$ spectral region (O'Dell et al., 1967), and from a rocket with an electronographic camera sensitive in the 1230-2100 region (Henry and Carruthers, 1970). On the Gemini photograph the nebula is unexpectedly bright, amorphous in appearance, with clear delineation of the amorphous nebulosity in its western extension. On the rocket photograph, however, there is no trace of the nebulosity:

Why did Henry and Carruthers fail to observe Barnard's Loop? An examination of their data (Weber et al., 1971) indicates the faintest source they could detect was a $V=8^{m} .2$ B9 star. From OAO photometry data we find an $8^{m} 2 \mathrm{~B} 9 \mathrm{~V}$ star has an average flux of $5.6 \times 10^{-12} \mathrm{erg} \mathrm{cm}^{-2} \mathrm{~s}^{-1} \AA^{-1}$ over the bandpass of their instrument (1230$2100 \AA$ ). Since this flux is distributed over a $2^{\prime}$ diameter image, their detection threshold for extended objects is $2.1 \times 10^{-5} \mathrm{erg} \mathrm{cm}^{-2} \mathrm{~s}^{-1} \AA^{-1} \mathrm{sr}^{-1}$.

By comparison, O'Dell et al. measured a surface brightness of $5.1 \times 10^{-8} \mathrm{erg} \mathrm{cm}^{-2}$ $\mathrm{s}^{-1} \AA^{-1} \mathrm{sr}^{-1}$ in one of the brightest parts of the Loop in their bandpass (2200-4900 $\AA$ ). Using OAO data to find the difference in grain albedo and illuminating flux at the two effective wavelengths, we find the nebula should have a surface brightness of

$$
\left(\frac{0.9}{0.4}\right)(2.9)\left(5.1 \times 10^{-8}\right)=3.3 \times 10^{-7} \mathrm{erg} \mathrm{cm}^{-2} \mathrm{~s}^{-1} \AA^{-1} \mathrm{sr}^{-1}
$$

at $1500 \AA$. This is a factor of 64 below the detection threshold of Henry and Carruthers.

Selected Area 97 is located in the same nebulosity 50' SSE of the point measured by O'Dell et al. (1967). Examining our OAO data, we find average scattered light intensities of $7.9 \times 10^{-8} \mathrm{erg} \mathrm{cm}^{-2} \mathrm{~s}^{-1} \AA^{-1} \mathrm{sr}^{-1}$ in the $2200-4900 \AA$ region, and $3.9 \times$ $10^{-7} \mathrm{erg} \mathrm{cm}^{-2} \mathrm{~s}^{-1} \AA^{-1} \mathrm{sr}^{-1}$ in the $1230-2100 \AA$ region. This is quite good agreement 
with O'Dell et al., well within their estimated error. We conclude the Barnard's Loop nebula has an ultraviolet surface brightness consistent with our albedo curve and O'Dell et al.'s measurements, and that Henry and Carruthers' camera was not sensitive enough to detect it.

\section{Summary}

Our preliminary analysis of OAO observations of the Merope and Barnard's Loop Nebulae shows the increase in the albedo of interstellar grains below $2000 \AA$ is real and occurs in individual reflection nebulae as well as the general interstellar medium. Henry and Carruthers (1970) did not observe Barnard's Loop in the 1230-2100 region because its surface brightness is far below the detection threshold of their camera. The OAO measurements of Barnard's Loop are in excellent agreenent with those of O'Dell et all. (1967).

A comparison of the albedo of grains in Merope and Barnard's Loop Nebulae with the albedo of grains in the general interstellar medium shows evidence of differences in the composition and size distribution of grains due to variations in the local radiation field. This difference is in the sense that the absorbing particles, and then the small scattering particles disappear as the intensity of radiation from nearby stars increases.

\section{Acknowledgements}

It is a pleasure to acknowledge the financial support furnished by NASA grant NGR 36-010-016 to Adolf N. Witt and NASA grant NGR 06-003-179 to Charles F. Lillie.

\section{References}

Code, A. D.: 1970 , private communication.

Code, A. D., Houck, T. E., Bless, R. C., McNall, J. F., and Lillie, C. F.: 1970, Astrophys. J. $161,377$.

Collins, O. C.: 1937, Astrophys. J. 86, 529.

Henry, R. C. and Carruthers, G. R.: 1970, Science 170, 527.

Hills, J. G.: 1972, Astron. Astrophys. 17, 155.

Lillie, C. F.: 1968, Bull. Am. Astron. Soc. 1, 198.

Lillie, C. F.: 1971, in A. D. Code (ed.), 'Scientific Results from the Orbiting Astronomical Observatory (OAO-2)', NASA SP-310.

O'Dell, C. R.: 1965, Astrophys. J. 142, 604.

O'Dell, C. R., York, D. G., and Henize, K. G.: 1967, Astrophys. J. 150, 835.

Smith, L. L., Roach, F. E., and Owen, R. W.: 1965, Planetary Space Sci. 13, 207.

Weber, S. V., Henry, R. C., and Carruthers, G. R.: 1971, Astrophys. J. 166, 543.

Witt, A. N.: 1973, this volume, p. 53.

Witt, A. N. and Lillie, C. F.: 1971, in A. D. Code (ed.), 'Scientific Results from the Orbiting Astronomical Observatory (OAO-2)', NASA SP-310. 